\title{
When may doctors give nurses telephonic treatment instructions?
}

\author{
D J McQuoid-Mason, BComm, LLB, LLM, PhD \\ Centre for Socio-Legal Studies, University of KwaZulu-Natal, Durban, South Africa
}

Corresponding author: D J McQuoid-Mason (mcquoidm@ukzn.ac.za)

\begin{abstract}
Doctors are expected to examine their patients before issuing telephonic instructions to nurses. However, in emergencies or when they are aware of the health status of their patients, it may be justified for a doctor to issue telephonic instructions to nurses without examining the patient. Doctors on call owe a special duty to patients, who they may have to examine or arrange for another doctor to do so before issuing telephonic instructions. In deciding whether doctors acted reasonably in issuing telephonic instructions to nurses, the courts will decide whether they exercised the same degree of skill and care as reasonably competent practitioners in their branch of the profession. Suggestions are made concerning doctors giving telephonic instructions to nurses regarding patients they have not examined.
\end{abstract}

S Afr Med J 2016;106(8):787-788. DOI:10.7196/SAMJ.2016.v106i8.10830

When is it legal for doctors to give nurses telephonic treatment instructions? Common sense indicates that good medical practice expects doctors to have examined the patient beforehand, except in emergencies or when they know the patient's health history. To answer whether these assumptions are consistent with the law we must consider: ( $i$ ) whether it makes a difference legally if telephonic instructions are regarded as telemedicine or not; (ii) what constitutes a medical emergency justifying telephonic instructions; (iii) whether a personal examination by the doctor is necessary; (iv) whether the situation changes if the doctor is 'on call'; and $(v)$ whether it is sufficient for doctors to issue instructions to nurses based on their patients' health histories.

\section{Does it make a legall difference if telephonic instructions constitute telemedicine?}

Telemedicine is defined as 'the practice of medicine, from a distance, in which interventions, diagnostic and treatment decisions and recommendations are based on clinical data, documents and other information transmitted through telecommunication systems. ${ }^{[1]}$ This definition which refers to 'other information transmitted through telecommunication systems' seems to cover telephonic treatment instructions to nurses by doctors. However, the HPCSA's Draft General Ethical Guidelines for Good Practice in Telemedicine state that 'Telemedicine generally does not include audio-only mechanisms, telephone conversations, e-mail, instant messaging conversations or faxes.'[2] Thus the HPCSA regards audio-only telephonic instructions such as those to nurses as falling outside the scope of the HPCSA Guidelines for Telemedicine. However, presumably face-to-face Skype-type conversations where the parties, including patients, are visible to each other might fall under the Guidelines for Telemedicine.

I submit that the courts will not dwell on whether audio-only mechanisms fall under the definition of telemedicine or not, but will consider what ought to be regarded as good medical practice for treatment instructions given over the telephone to nurses. The test will be whether the treating doctors have exercised the same degree of skill and care as reasonably competent practitioners in their branch of the profession when giving the telephonic instructions. ${ }^{[3]}$ Judges will then usually follow what the medical profession regards as good medical practice, ${ }^{[4]}$ but are not obliged to do so. ${ }^{[3]}$

\section{What constitutes an emergency justifying telephonic instructions?}

The Constitutional Court has defined a medical emergency as a dramatic, sudden situation or event which is of passing nature in terms of time ${ }^{[5]}$ that can be cured through medical treatment. Therefore emergency medical treatment refers to conditions that can be rectified through medical treatment - unlike chronic illnesses that cannot be cured. ${ }^{[5]}$

Emergency medical treatment is needed when patients are faced with the possibility of death, serious bodily injury or deterioration in health. ${ }^{[6]}$ Therefore, if in such circumstances the doctors cannot reach the patients in time for an examination, it is reasonable for them to issue telephonic instructions to the relevant nurses to stabilise the patients until examined by the instructing doctor or another doctor.

If the patient is mentally able to give consent or a proxy is available to do so in terms of a court order or the provisions of the National Health Act, ${ }^{[7]}$ emergency medical treatment should be given after obtaining such consent. The following may act as proxies in order of preference: a court-appointed curator, or the spouse or partner, parent, grandparent, adult child or sibling of the patient. ${ }^{[7]}$ Alternatively, persons appointed by patients in writing before becoming mentally incapacitated may be authorised to give consent. ${ }^{[7]}$ If the patient or a legal proxy cannot give consent, emergency treatment may be given without consent - provided it is not against a previous directive issued by a patient refusing treatment, e.g. a refusal to accept a blood transfusion for religious reasons. ${ }^{[8]}$

Before issuing telephonic instructions doctors should consider whether telephone management is appropriate in the situation concerned, and reflect on this throughout the call. ${ }^{[9]}$ To issue telephonic instructions to nurses in emergencies, a doctor should: (i) request and record the name and surname of the nurse; (ii) obtain a detailed history of the patient's health and condition from the nurse; (iii) paraphrase and reflect back to the nurse the nurse's description of the patient's condition; (iv) give the nurse the instructions and ensure that they are understood; $(v)$ get the nurse to repeat the instructions; (vi) repeat the instructions to another nurse if available and record 
her or his name and surname; and (vii) if a second nurse is not available repeat the instructions to the first nurse and get her or him to repeat them. This protocol is based on suggested guidelines for when doctors telephonically give instructions to patients, ${ }^{[9]}$ which I have adapted to apply to telephonic instructions to nurses.

\section{Is a personal examination by the doctor necessary before issuing telephonic instructions to nurses?}

Doctors should always consider whether issuing telephonic instructions without examining the patient is appropriate in the circumstances concerned, and should review their decision throughout. ${ }^{[9]}$ Doctors must be satisfied that it is in the patient's best interests to issue treatment instructions to nurses without having examined the patient.

The UK General Medical Council (GMC) has stated that it may not be appropriate to issue telephonic instructions where: $(i)$ the patient is not previously known to the doctor; (ii) the assessment may be helped by examination of the patient; and (iii) there is no provision for appropriate monitoring of the patient or follow-up care. ${ }^{[10]}$ Although the GMC later withdrew this statement, the South African courts may find that it is in line with common sense and good medical practice, and provides useful guidelines for local doctors when deciding whether or not to issue telephonic instructions to nurses.

\section{Does the situation regarding telephonic treatment instructions change if the doctor is 'on call'?}

Whether or not 'on call' will make no difference to doctors' legal liability should they have failed to act as a reasonably competent doctor ought to have acted under similar circumstances. ${ }^{[3]}$ Doctors 'on call' should follow the proposed protocols when issuing telephonic instructions. However, if the nurses' information indicates that patients must be examined by a doctor before instructions are issued, the doctor 'on call' must either examine the patient personally or arrange for another medical practitioner to do so. ${ }^{[10]}$ This applies whether the doctor 'on call' is on or off the premises of the health institution concerned at the time the nurses seek treatment guidance. The test for legal liability will be whether a reasonably competent doctor 'on call' would have examined the patient, or have arranged for another doctor to do so, before issuing the telephonic instructions; or whether a reasonably competent doctor would have considered it reasonable to issue treatment instructions to the nurses without examining the patient. ${ }^{[3]}$

\section{May doctors issue instructions to nurses based on their patients' health histories?}

Before issuing telephonic instructions a doctor should always consider whether telephone management is appropriate in the situation concerned, and reflect on this throughout the call. ${ }^{[9]}$

\section{Mentally competent patients}

Despite doctors being aware of their patients' health histories, where they are mentally competent it may be necessary to clarify their condition by telephonically speaking with the patient before issuing instructions to the nurses. When able to speak telephonically to the patient before instructing the nurses the doctor should: (i) obtain and record a history of the patient's condition since last examined by the doctor; (ii) give the patient treatment options; (iii) obtain an informed consent regarding the treatment; (iv) give specific advice on follow-up; and $(v)$ ask the patient to repeat the advice given to check that the consent is informed. ${ }^{[9]}$

As in emergencies, the doctor should then instruct the nurse regarding the treatment of the patient and: (i) request and record the name of the nurse; (ii) give the nurse the instructions and ensure that they are understood; (iii) get the nurse to repeat the instructions; (iv) repeat the instructions to another nurse, whose name and surname should be recorded; and $(v)$ if another nurse is not available repeat the instructions to the first nurse and get her or him to repeat them. ${ }^{[9]}$

\section{Mentally incompetent patients}

Where the patient is mentally incompetent and a legal proxy is available in terms of the National Health Act, ${ }^{[7]}$ and has knowledge of the patient's condition since the doctor last examined the patient, the steps applied to a telephonic consultation with a mentally competent patient could be applied to the proxy - save that the first step should be to record the name, identity number and telephone number of the proxy and the basis on which he or she is acting as a proxy.

Where the patient is not mentally competent and no proxy is available, the same steps should be followed as in the case of emergency medical treatment.

\section{Conclusion}

Nurses may sometimes fail to carry out the directions of doctors accurately or effectively. Therefore, doctors should be cautious about issuing telephonic instructions and ensure that they are clearly understood. Accurate records of the instructions given must be kept by the doctors and nurses to prevent ambiguities arising should patients exposed to telephonic instructions suffer harm as a result of these not being properly carried out. Provided doctors are satisfied that telephonic instructions to nurses are appropriate in a particular situation without examining the patient, they should follow the proposed protocols for emergency and non-emergency treatment of patients.

The law courts will decide whether the doctor in a particular situation has exercised the degree of skill and care of a reasonable medical practitioner in their field of expertise. Although the courts will generally follow what the medical profession regards as good medical practice they are not bound to do so. Hopefully these protocols will assist doctors and nurses to avoid unprofessional conduct that may harm their patients when doctors give, and nurses receive, telephonic instructions.

\footnotetext{
1. World Health Organization. Statement on Accountability, Responsibility and Ethical Guidelines in the Practice of Telemedicine. Geneva: WHO, 2005:Preamble para 1.

Health Professions Council of South Africa. Draft General Ethical Guidelines for Good Practice in Health Professions Council of South Africa.
Telemedicine. Pretoria: HPCSA, 2014:para 3.

3. Cf. Castell v De Greef 1993 (3) SA 501 (C).

3. Cf. Castell v De Greef 1993 (3) SA
4. Van Wyk v Lewis 1924 AD 438.

4. Van Wyk v Lewis $1924 \mathrm{AD} 438$.

5. Soobramoney v Minister of Health, KwaZulu-Natal 1998 (1) SA 765 (CC) 778.

6. Strauss SA. Doctor, Patient and the Law. 3rd ed. Pretoria: JL van Schaik, 1991:93.

Republic of South Africa. Section 7(1) of the National Health Act No. 61 of 2004. Pretoria: Government Gazette, 2004.

8. ES v AC [2015] NASC 11. www.saflii.org/na/cases/NASC/2015/11.html (accessed 20 March 2016). 9. Cf. Chloe Borton. Telephone Consultations, 12 June 2009. http://patient.info/doctor/telephone -consultations (accessed 17 March 2016).

10. General Medical Council. Providing Advice and Medical Services Online or by Telephone, 1998 2004. London: General Medical Council, 1998-2004. www://gmc-uk.org/providing_advice_nov1998. pdf_25416215.pdf (accessed 17 March 2016).
} 